\title{
PRÁCTICAS DE CUIDADO NUTRICIONAL INFANTIL, REPRESENTACIONES DE CUIDADORES NUTRICIONALES Y EFECTORES DE SALUD
}

Scruzzi Graciela Fabiana ${ }^{1}$ Lucchese Marcela²

Lic. en Nutrición, Especialista en

Epidemiologia. Docente de la Universidad Nacional de Córdoba y de la Universidad Católica de Córdoba, Argentina. Correo gracielafabiana@hotmail.

com

2Doctora en Ciencias de la Salud. Licenciada y Profesora en Ciencias de la Educación. Docente de la Universidad Nacional de Córdoba y de la Universidad Católica de Córdoba, Argentina

Trabajo recibido: 22 de mayo de 2016.

Aprobado: 16 de septiembre de 2016.

\section{CHILD NUTRITIONAL CARE PRACTICES, REPRESENTATIONS OF NUTRITIONAL CA- TETAKES AND HEALTH EFFECTORS}

PRÁTICAS DE CUIDADOS NUTRICIONAIS INFANTIS, REPRESENTAÇÕES DE CUIDADORES NUTRICIONAIS E AGENTES DE SAÚDE

\section{Resumen}

Objetivo: Indagar las representaciones de los cuidadores nutricionales y los efectores de salud acerca de las prácticas de cuidado nutricional infantil (PCNI) consideradas por los programas y planes de salud implementados en la ciudad de Córdoba.

Método: Investigación cualitativa. Se utilizaron entrevistas en profundidad explorando conocimientos, creencias y actitudes sobre PCNI, participaron 19 cuidadores de niños bajo programa Control de Crecimiento y Desarrollo y 6 efectores de salud de Córdoba. El análisis de la información se efectuó a través de la teoría fundamentada.

Resultados: El cuidado es entendido y asumido por cuidadoras y efectores de manera diversa, generando encuentros y desencuentros entre los distintos actores; se visualizan problemáticas en la coordinación y comunicación acerca de las prácticas de cuidado nutricional entre los actores, desarrollándose en un escenario marcado por la hegemonía; el cuidado es considerado un rol femenino, con escasa participación del varón. 
Conclusiones: Las representaciones están dominadas por el modelo hegemónico imperante en salud. Las cuidadoras se sitúan como tales, como mandato natural por ser mujer. Sería necesario transitar del modelo hegemónico hospitalocéntrico a otro, para que los profesionales se acerquen y trabajen con la comunidad.

Palabras clave: Representaciones, Cuidados Nutricionales, Infancia.

\section{Abstract:}

Objective: Inquire representations of nutritional caretakers and health effectors about practices of child nutritional care (PCNC) considered by health plans and programs implemented in the city of Cordoba.

Method: Qualitative research. In-depth interviews were used exploring knowledge, believes and attitudes about PCNC, participants were 19 caretakers of children under the program Growth and Development Control and 6 health effectors from Cordoba. Information analysis was performed using grounded theory.

Results: Care is understood and assumed by caretakers and effectors in different ways, generating agreements and disagreements among the different actors; problems in the coordination and communication about nutritional care among actors are observed; thus developing a setting marked by hegemony; care is considered a female role with very little male participation.

Conclusions: Representations are dominated by the hegemonic model prevailing in health. Caretakers are self-placed as such, as a natural law for being women. It would be necessary to move from the hegemonic hospital-centered model to another, so that professionals may come closer and work with the community.

Key Works: Representations; Nutritional Care; Childhood.

\section{Resumo:}

Objetivo: explorar as representações de cuidadores nutricionais e agentes de saúde sobre as práticas de cuidado nutricional infantil (PCNI) considerados pelos programas e planos de saúde conduzidos na cidade de Córdoba.

Método: Pesquisa qualitativa. Entrevistas em profundidade foram usadas para explorar o conhecimento, crenças e atitudes sobre PCNI, participaram 19 babás sob o programa de Controle do Crescimento e Desenvolvimento e 6 agentes de saúde de Córdoba. A análise dos dados baseou-se na teoria fundamentada.

Resultados: O cuidado é compreendido e aceito pelos cuidadores e agentes de diferentes maneiras, gerando--se encontros e divergências entre os vários intervenientes; Evidenciam-se questões na coordenação e comunicação sobre práticas de cuidados nutricionais entre as partes interessadas, desenvolvendo-se em um cenário atravessado pela hegemonia; os ditos cuidados consideram-se um papel próprio do sexo feminino, com pouco envolvimento dos homens.

Conclusões: As performances são dominadas pelo modelo hegemônico vigente na área de saúde. As cuidadoras são posicionadas como tais, como dispondo de um mandato natural por serem mulheres. Seria necessário mudar do modelo hospitalocêntrico hegemônico para outro a fim de os profissionais se aproximarem da comunidade e trabalharem com ela.

Palavras-chave: Representações, Cuidados Nutricionais, Infância. 
En este estudio se abordan las prácticas de cuidado nutricional infantil (PCNI) consideradas por los programas y planes de salud implementados en la ciudad de Córdoba, 2013, para lo cual se efectúa un abordaje de las prácticas de cuidado como eje de la investigación y un abordaje de las representaciones de los cuidadores y efectores sobre las prácticas de cuidado en los programas de salud y en su implementación.

Se definen las prácticas de cuidado nutricional infantil como "el entrecruzamiento de las formas socialmente aprendidas de resolver cotidianamente la selección, preparación y distribución de los alimentos como parte de las estrategias de consumo de las familias y las acciones de los adultos encaminadas a orientar el desarrollo de los niños, vinculadas a la crianza y cuidado de ellos" (1).

El abordaje de las PCNI abarca aspectos como: nutrición en el embarazo, lactancia materna, alimentación complementaria oportuna, selección, preparación y distribución de alimentos, dentro de éste se contemplan algunas dimensiones como: cantidad y consistencia, otro aspecto de este abordaje refiere a las pautas de crianza y dentro de ellas el vínculo que se da entre el/la cuidador y el niño en el acto del comer (2).

Habitualmente, el cuidado se considera una actividad que realizan las mujeres en el ámbito privado, dentro del cuidado, las prácticas de selección, preparación, distribución de los alimentos constituyen aspectos claves para la nutrición infantil $(1,3,4)$.

El desarrollo de la primera infancia, en particular el desarrollo físico, socioemocional y lingüístico-cognitivo, determina de forma decisiva las oportunidades en la vida de una persona y la posibilidad de gozar de buena salud, pues afecta la adquisición de competencias, educación y oportunidades laborales. Eso tiene enormes consecuencias para su salud y para la sociedad en su conjunto.

Desde Unicef el cuidado se define como la provisión a nivel familiar y comunitario de tiempo, atención y apoyo para satisfacer las necesidades físicas, mentales y sociales de los niños y niñas en crecimiento y otros miembros del hogar. La ingesta de nutrientes, la salud y el desarrollo cognitivo y psicosocial de niños se ven afectados particularmente por el conjunto de comportamientos implementados por los cuidadores para la satisfacción de estas necesidades (4).

Las prácticas alimentarias de los niños y niñas de menores de 5 años de edad, dependen en gran medida de los cuidadores nutricionales -que son casi exclusivamente las mujeres, madres- responsables de la selección, preparación y distribución de los alimentos al interior del hogar y del establecimiento de las pautas que se ponen en juego en el momento mismo de la alimentación, acompañadas en muchos caso por la familia extendida (abuelo/ as, tío/as, hermano/as) y en menor medida por organizaciones o instituciones del entorno próximo (4).

Según lineamientos de FAO (Organización de las Naciones Unidas para la Alimentación y la Agricultura) las prácticas de cuidado nutricional constituyen el menos estudiado de los tres factores subyacentes que determinan la ingesta de nutrientes y la salud y por tanto, la supervivencia infantil, el crecimiento y el desarrollo, según el modelo conceptual de Unicef. Para los otros dos factores subyacentes seguridad alimentaria familiar y salud, existen numerosas investigaciones que los vinculan con el estado nutricional (5). 
Autores como Letablier y Aguirre consideran que las problematizaciones en torno a las prácticas y relaciones de cuidado deben analizarse en las intersecciones del espacio doméstico con las políticas públicas y con otras formas mercantilizadas o comunitarias de cuidado que tienen lugar en nuestra sociedad $(6,7)$.

Se asume desde los gobiernos que el cuidado es una responsabilidad fundamentalmente de los hogares, y la provisión pública es simplemente un complemento para aquellos hogares que no pueden resolverlo por sí mismos (8).

EnArgentina, históricamente las políticas estatales en torno al cuidado, se complementaron con las acciones desarrolladas por las familias en el marco de la consolidación del modelo de familia nuclear. Así la atención de las necesidades de cuidado recayó principalmente sobre las unidades domésticas consolidando un modelo familista y feminizado de cuidado infantil, en tanto las prácticas de cuidado fueron asumidas principalmente por las familias y al interior de las mismas fundamentalmente por las mujeres (9).

En relación a la implementación de las políticas en la niñez, el Estado argentino ha avanzado en el reconocimiento del derecho de los niños y las niñas al cuidado y la educación desde temprana edad: incorporación en la Constitución, en el año 1994 de los tratados internacionales sobre niñez y creación de la Ley Nacional 26.061 de Protección Integral de los Derechos de las Niñas, Niños y Adolescentes (10).

El enfoque de derechos en las políticas y estrategias de desarrollo, considera que el marco conceptual que brindan los derechos humanos como derechos legitimados por la comunidad internacional, ofrece un sistema coherente de principios y pautas aplicables en las políticas de desarrollo (8).Incluir la perspectiva de derechos en el diseño de políticas públicas consiste en revisar no sólo sus contenidos u orientación, sino también los parámetros necesarios para su evaluación y fiscalización.

A partir de los aspectos presentados y la importancia de la nutrición en los niños como futuros ciudadanos; el presente trabajo pretende tomar como eje de estudio las prácticas de cuidado nutricional infantil como una forma de analizar las mismas, con el objeto de contribuir en relación al tema a quienes tienen la decisión a nivel de estado.

En relación al espacio doméstico, así como a las formas comunitarias de cuidado, una manera de interpretarlas es a través de las representaciones sociales. Éstas son construidas a partir de procesos de interacción y de comunicación social, y se cristalizan en prácticas sociales; quedando plasmados en éstas los aspectos sociales, culturales e históricos.

El presente estudio toma la vertiente procesual de Moscovici, quien define la representación como un corpus organizado de conocimientos y una de las actividades psíquicas gracias a las cuales los hombres hacen inteligible la realidad física y social, se integran en grupos y promueven relaciones de intercambio. Se puede reconocer en ella la presencia de estereotipos, opiniones, creencias y valores que orientan las actitudes de los sujetos, constituyéndose en códigos, lógicas y modos de interpretación. El autor pone el acento en la función de contribuir en la formación de conductas y en la orientación de las prácticas sociales (11). La línea procesual parte de la complejidad de las representaciones y es desarrollada por Jodelet y Moscovici, desde una vertiente constructivista. Se privilegian desde este enfoque dos vías de acceso al conocimiento, una a través de análisis cualitativos y otra a través de la triangulación $(12,13)$.

En la presente investigación, se indagaron las representaciones, intentando determinar qué se conoce (información), qué se cree o cómo se interpreta (creencias y significados) y cómo se orienta la conducta (actitud) desde una concepción de sujeto activo inserto en un medio complejo (11). 
En este marco, el objetivo de trabajo fue Indagar las representaciones de los cuidadores nutricionales y los efectores de salud acerca de las prácticas de cuidado nutricional infantil consideradas por los programas y planes de salud implementados en la ciudad de Córdoba.

\section{Material y métodos}

Se trata de un estudio de carácter cualitativo orientado a explorar el significado que los actores les atribuyen a las prácticas de cuidado nutricional infantil (11).

Para indagar las representaciones de los efectores de salud y los cuidadores nutricionales acerca de las prácticas de cuidado nutricional infantil consideradas por los programas y planes de salud implementados en la ciudad de Córdoba, se realizaron entrevistas en profundidad a efectores y cuidadores en dos centros de salud municipales, así como a efectores de programas provinciales (Maternidad e Infancia y Plan Nacer), a fin de considerar las diferentes perspectivas de las prácticas de cuidado nutricional; la información se analizó mediante la Teoría Fundamentada y se efectuó triangulación de los datos.

La indagación de las representaciones, en este trabajo se centró en la vertiente procesual de Moscovici, que está orientada hacia una postura socio-constructivista. Moscovici reconoce 3 componentes fundamentales en las mismas (información, creencias y actitudes) (11).

La entrevista en profundidad es un proceso de organización de los hechos y representaciones de la conducta a través de un diálogo social donde sus integrantes interaccionan con el objetivo de crear una relación dinámica donde los temas se van generando libremente $(14,15)$.

Para la selección de los cuidadores, se trabajó con un muestreo teórico que consiste en el proceso de recolección de datos para generar una teoría por la cual el investigador, conjuntamente, selecciona, codifica y analiza su información y decide cuál escoger. Para la selección de efectores, se trabajó con un muestreo intencional que consiste en un esfuerzo deliberado de incluir grupos típicos.

Los sujetos, objeto de estudio, fueron 1 efector del Plan Nacer, 1 efector de Maternidad e Infancia, 4 efectores de dos centros de salud municipales y 19 cuidadores de los niños que asistieron al programa Control de Crecimiento y Desarrollo (CCyD) implementado en los centros de salud municipales. Se trabajó hasta la saturación. La saturación implica el límite a partir del cual cesa el muestreo, y refiere a la imposibilidad de que un grupo de categorías siga brindando información relevante.

El análisis se efectuó de acuerdo a la Teoría Fundamentada, en tal sentido, Glaser y Strauss (1967) proponen estrategias principales para desarrollar teoría fundamentada. Dentro de esta línea, en el presente trabajo se utilizó el Método Comparativo Constante (MCC), por el cual el investigador, simultáneamente, codifica y analiza datos para desarrollar concepto. Mediante la comparación constante de incidentes específicos de la información, el investigador refina conceptos, identifica propiedades, explora interrelaciones e integra la misma en una teoría coherente (16).

\section{Resultados}

A partir de la indagación de información, creencias y actitudes, se elaboraron las siguientes categorías de análisis. 


\section{a) El cuidado: Encuentros y Desencuentros entre las representaciones de efectores y de cuidadoras.}

Esta categoría se organiza en los siguientes aspectos:

- El cuidado en términos de información recibida y prácticas vinculadas al cuidado.

- La relación entre los saberes médicos y los saberes cotidianos de los cuidadores en las prácticas de cuidados

Si entendemos al cuidado como la provisión a nivel familiar y comunitario de tiempo, atención y apoyo para satisfacer las necesidades físicas, mentales y sociales de los niños y niñas en crecimiento y otros miembros del hogar (4), entonces esta provisión dependerá de los conocimientos y creencias que se tenga acerca del cuidado. Por lo tanto el apoyo a los cuidadores es esencial, ya que en parte, la información brindada acerca de algunos aspectos de cuidado como lactancia materna e introducción de alimentos (17), se constituyen en factores que contribuyen al crecimiento y desarrollo de los niños, según Unicef.

De los relatos de cuidadoras y de efectores de los centros de salud se observa concordancia en cuanto a la información brindada sobre el cuidado nutricional infantil. Tanto las cuidadoras entrevistadas como los efectores refieren que la información gira en torno a los beneficios de la práctica de lactancia materna y etapas en la alimentación del niño, que en términos de Engle P, Menom P y Haddad, se vinculan a algunos aspectos del cuidado. Asimismo, las cuidadoras expresan que no recibieron información respecto de las dimensiones como son cantidades, formas de preparación y distribución, pautas de crianza, resolución de problemas en torno a la alimentación en los primeros años.

De las narraciones de las cuidadoras y los efectores se observa que la asistencia en consultorio se convierte en el único lugar de información, como una práctica en forma reiterada, aspecto que está en tensión con la propuesta de los planes y programas de salud implementados en la ciudad de Córdoba, desde los cuales se plantea la necesidad de un trabajo en la comunidad (17).

Los relatos de las cuidadoras dan cuenta de que la información en torno a la alimentación, dentro de la consulta es entendida como un hecho prescriptivo, tal como lo describen Purnell y Paulanka y lo retoman Melguizo y Alzate (18); los efectores también reconocen que no se detienen a preguntarles su opinión o sus sentimientos en un espacio dialógico, la información se brinda de manera individual y unidireccional dentro de la consulta; no se identifica en los relatos, tanto de cuidadores como efectores, la presencia de espacios grupales de reflexión, donde la comunicación se pudiera dar de manera más horizontal, como proponen Aguiló y Cucco (19) y las madres podrían compartir con pares sus problemáticas en torno al cuidado nutricional infantil; tal como lo proponen los planes y programas del Ministerio de Salud (17).

“Me enseñaban cómo preparar la leche, cuántas medidas". (Entrevista 1, 42 años, familia nuclear, no comparte la crianza, Centro de Salud 2)

"Más que nada sobre que tenía que comer cada mes". (Entrevista 1, 27 años, familia nuclear, no comparte la crianza, Centro de Salud 1)

Definidas las Prácticas de Cuidado Nutricional Infantil como las formas socialmente 
aprendidas de resolver el cuidado de niños y niñas, es necesario conocerlas e indagar las fuentes de conocimiento (1). Se advierte en las cuidadoras que si bien no cuestionan las recomendaciones ofrecidas por el efector del centro de salud en torno a las prácticas de cuidado nutricional infantil, la principal fuente que ellas expresan, refiere al saber práctico que circula entre sus familiares, quedando estas prácticas, muchas veces invisibilizadas ante el discurso legitimado de los profesionales de salud; quienes no indagan sobre las prácticas de cuidado que llevan adelante las cuidadoras (20). Esta situación evidencia desencuentros en el proceso de información, debido a que se establece una forma de relación asimétrica entre los saberes médicos y los saberes cotidianos de los cuidadores. Si se reconoce que la provisión de cuidado depende de los conocimientos y creencias acerca del cuidado, por lo tanto no resulta de menor importancia el reconocimiento de los saberes cotidianos de los cuidadores por parte del equipo de salud, ya que éste es un aspecto esencial en la provisión de cuidado según Engle, P., Menom, P., Haddad (4, 5).

"Lo de la teta no, porque a mi bebe cuando nació bajó de peso, entonces en el hospital le dieron biberón y cuando lo quise prender al pecho no había caso". (Entrevista 1, 27 años, familia nuclear, no comparte la crianza, Centro de Salud 1)

“Todo bien, yo lo tomo como una guía por más que mi mamá no esté de acuerdo, por ejemplo ella se asusta cuando yo hago dormir a mi bebé boca arriba, antes te decían que tenía que ser en otras posiciones." (Entrevista 3, 36 años, familia nuclear, comparte la crianza con la abuela, Centro de Salud 1)

“(...) antes nos criaban con té de manzanilla para los dolores de panza y con aspirinetas. Ahora la pediatra me dice que no le dé para nada aspirinetas, no sé si cambió la composición". (Entrevista 3, 36 años, familia nuclear, comparte la crianza con la abuela, Centro de Salud 1)

Toda práctica, como forma socialmente aprendida, está influenciada por las creencias. Las creencias son algo distinto de aquello que conocemos o sabemos sobre algo. Aunque alguien no pueda explicar la lógica de aquello que acepta como verdad, o aunque no posea muchos conocimientos al respecto, la fe en aquello que cree lo lleva a actuar en consecuencia aun reconociendo que el carácter dinámico y flexible de las creencias de los seres humanos no permite predecir las prácticas. Y si no se actúa consecuentemente con aquello que creemos se experimenta distintos grados de malestar o incertidumbre (18). Esto se observa en los relatos de las cuidadoras que ponen en tensión la información que reciben de los profesionales con la de su propio entorno, quedando despojadas de saber, sintiéndose inseguras y dependientes del saber científico.

"Me explicaron que lo ideal es que tome la teta, pero él toma teta y mamadera, este va a ser el segundo control”. (Entrevista 4, 22 años, familia extendida, comparte la crianza con la abuela, Centro de Salud 2).

"Y está bien ellos saben, la puse en práctica pero no había caso Andrés lloraba lloraba escupía la comida y terminaba dándole la leche". (Entrevista 1, 42 años, familia nuclear, no comparte la crianza, Centro de Salud 2)

La actitud asumida por el equipo de salud al aproximarse al paciente en la relación médico-paciente, está condicionada por el modelo teórico en el cual haya sido formado, aunque en la mayoría de los casos este modelo no se haya hecho explícito durante su 
formación. Desde el modelo hegemónico se espera del médico que actúe con eficiencia e idoneidad en la resolución de problemas de salud, donde la comunicación se da de manera asimétrica, autoritaria, el paciente tiene una participación subordinada y pasiva, y por lo tanto su conocimiento es excluido; desde este modelo los procesos sociales, culturales o psicológicos son anecdóticos por lo tanto no forman parte de la comprensión de la situación por parte del médico (21), en relación al análisis que se efectúa en esta categoría respecto a la información y su vinculación con los cuidadores, se observa este tipo de relación médico-paciente de forma frecuente entre los efectores y cuidadores de esta investigación, en contraposición a otros autores como Moore y otros, que plantean que la comunicación efectiva se caracteriza por asegurar una interacción, en vez de una transmisión directa o simple entrega de información (22).

\section{b) Respecto de la cobertura en la atención de salud}

Todos los países aspiran a que sus ciudadanos puedan gozar de la mayor calidad de vida posible, en los últimos años ha emergido un consenso en el ámbito internacional sobre la importancia de garantizar la salud. Se destaca la importancia de la cobertura universal en salud mediante mecanismos de APS y protección social. Los sistemas de salud no sólo deben contribuir a mejorar la salud de la población, sino también deben contribuir a la cohesión social (23). El programa CCyD no es un programa aislado, sino que se encuentra inmerso dentro de un conjunto de prestaciones que brindan los Centros de Atención Primaria de Salud; es por esto que se indagó acerca de la utilización, por parte de las cuidadoras, de los diferentes programas ofrecidos por los Centros de Salud, así como la valoración del funcionamiento del centro que realizan tanto cuidadoras como efectores.

A nivel central, los efectores refieren que existen herramientas (vistas domiciliarias, talleres de promoción, educación para la salud) dentro de cada programa para poder brindar información a cuidadores, y que la decisión respecto a la implementación de la misma está en cada centro de salud.

Los efectores relatan que la demanda de asistencia, por parte de la propia comunidad, dentro del consultorio, tanto para el Programa CCyD como los demás programas implementados (Control de Embarazo, Adulto Asintomático, Salud Sexual y Reproductiva, entre otros), no les permite desarrollar otras actividades, propuestas en los programas (17), las cuales se orientan a mejorar la calidad de la atención a la salud.

“yo no puedo hacer más que los controles, ¿que sería lo ideal?, que pudiéramos usar la sala de espera, y por ahi no lo podemos hacer porque nos supera lo asistencial, tenés 20 pacientes con fiebre afuera esperándote, por ahi es una falencia del sistema en realidad, se supone que nosotros en atención primaria deberíamos dedicarnos a esto a dar charlas a sensibilizar a educar y no a ver chicos con fiebre y como no tienen adonde lo vean, terminan cayendo todos acá, por ahí el tema asistencial nos supera" (Entrevista Efector Centro de Salud 1)

"Yo pienso que es posible en tanto y cuanto, cada equipo estuviera completo, si tenemos recursos vas a poder salir a la comunidad, porque vas a dejar trabajando un equipo en el centro y otro equipo afuera, si no, no podes salir a la comunidad...." (Entrevista Efector Centro de Salud 2)

En el presente trabajo se advierte que los centros de salud analizados siguen desarrollando 
la mayoría de sus actividades en el marco de la asistencia, observándose una escasa o nula incorporación de acciones de promoción, y de participación ciudadana. Cuestiones que tensionan los objetivos de los diferentes programas de salud en la ciudad de Córdoba. En este marco, Llobet considera que las prácticas institucionales ponen en tensión el objetivo de cuidado con la necesidad de reproducción de la institución, y con los postulados más generales de inclusión y socialización de las nuevas generaciones, por lo que se observa que la transformación de las concepciones sobre infancia generadas en las nuevas normativas a nivel central, no acompañan necesariamente las transformaciones institucionales en contextos específicos (24).

\section{c) La comunicación entre cuidadoras y efectores se desarrolla en un escenario marcado por la hegemonía}

El proceso de comunicación se encuentra en la génesis y en las funciones de las representaciones sociales y, desde luego, en su transmisión y difusión. Son mutuamente interdependientes, al punto en que cualquier consideración de las representaciones sociales también significa una consideración de la comunicación. Las representaciones sociales se originan en la comunicación, se manifiestan en ella y le confieren su influjo (15).

Quienes trabajan en salud no siempre se identifican en el rol de comunicadores sociales. En algunas situaciones los profesionales de salud son emergentes de un sistema que sigue basado en el modelo médico hegemónico y que aún padece una fragmentación y una desarticulación históricas. Así, desde ese paradigma, se piensa a la comunicación como la asimétrica relación "médico-paciente", donde el primero debe traducir para que el segundo obedezca (25).

Los efectores en sus relatos manifiestan que las cuidadoras toman la información brindada por ellos como un asesoramiento, confiando en el saber que los profesionales les proporcionan, a su vez expresan que no hacen una auto reflexión acerca de la consejería.

"Lo toman como una consejería como una herramientas más de los profesionales, me parece, la verdad que nunca me detuve a preguntarles a ver lo que sienten, por ahí cada uno, cada uno interpreta lo que puede, lo que quiere." (Entrevista Efector, Centro de Salud 2)

Las cuidadoras en sus relatos reconocen los conocimientos del equipo de salud y sólo aceptan lo expresado por médico sin cuestionarlo.

"Era como una orden (...) Porque la pediatra, me repetía siempre lo mismo y me decía media enojada qué tenía que hacer con mi bebe". (Entrevista 6, 21 años, familia nuclear, no comparte la crianza, Centro de Salud 1)

"No, yo no tenía mucha idea de estas cosas. (...) La información que recibí me era muy útil." (Entrevista 1, 27 años, familia nuclear, no comparte la crianza, Centro de Salud 1)

"La doctora me explicó que no le tengo que dar nada concentrado (...) va ella me dice, pero yo no le pregunto porque si o porque no, ella es la que sabe." (Entrevista 9, 38 años, familia nuclear, no comparte la crianza, Centro de Salud 1)

Las decisiones cotidianas que las familias toman sobre el cuidado y la educación de la/ os hija/os se vincula con procesos más amplios de producción social. Las personas, en el intercambio con otros, comparten una información que luego codificarán, ordenarán 
e interpretarán de acuerdo a sus creencias y a sus vivencias otorgándole un significado particular que influirá en las actitudes que asuman al respecto. De acuerdo a lo planteado por Mora, las representaciones sobre el cuidado, surgen desde este encuadre comunicativo que se establece entre las familias, la comunidad y el equipo de salud, por lo que es de importancia la articulación de estos aspectos para la comprensión de los saberes cotidianos de las cuidadoras (15), aspectos que no se observan necesariamente en el encuadre comunicativo entre los efectores y cuidadores.

Las cuidadoras que relatan haber tenido algún inconveniente con la alimentación del niño, refieren que desde el centro de salud les respondían que insistieran con la práctica que le proponían y si no podía hacerlo continuaban pidiendo que insistieran y algunas veces las retaban, lo que podría ser interpretado como una imposición(18).

"Me decía que insistiera (...) No, solo que insistiera." (Entrevista 6, 21 años, familia nuclear, no comparte la crianza, Centro de Salud 1)

"La pediatra me decía que insistiera y que lo prendiera a la teta, pero aun así no hubo forma, no quería”. (Entrevista 7, 27 años, familia monoparental, no comparte la crianza, Centro de Salud 1)

En estas expresiones se puede observar cómo, parte del saber femenino en salud, es resultado del conocimiento hegemónico de salud, consecuencia de un proceso de profesionalización. Según Hernández Tezoquipa, Arenas y Santiago, el desconocimiento de los saberes de otros y los conocimientos vistos como verdad se establecen en un tipo de relación que resultan producto de las relaciones de poder (26).Así, esta relación modelo médico hegemónico en la comunicación deviene de un proceso social más amplio y que requiere de su revisión, ya que la salud como objeto complejo (25) es la resultante de la interacción de factores sociales, económicos, culturales y ambientales, a fin de atender a las propuestas de los documentos oficiales, respecto a la concepción de salud y al enfoque de derechos (10).

\section{d) Las familias y el cuidado: Rol femenino, con escasa participación del varón}

Jodelet plantea que, dentro de las funciones sociales que cumple la representación, está la de orientar la conducta de los individuos, guiar las relaciones sociales y las comunicaciones sociales. Afirma que es importante conocerlas "para entender cómo la gente actúa en su vida, hay que ver cuál es el significado que la gente pone en su universo". Esta categoría se centra en las conductas de los proveedores del cuidado (11).

Las cuidadoras entrevistadas fueron en su totalidad mujeres madres, con edades comprendidas entre los 19 y 42 años y tienen entre 1 y 2 hijo/as. En cuanto al tipo de familia hubo diferencias según centro, mientras en el Centro de Salud 1 la mayoría vive con su pareja y no trabaja fuera del hogar; en el Centro de Salud 2 la mayoría conforma familias extendidas y no trabaja fuera del hogar. Las cuidadoras de ambos centros de salud que comparten la crianza, lo hacen con abuelas y tías y en un casi con el padrino del niño. Algunas mujeres madres mencionan que la participación del padre en temas referidos al cuidado del niño es escasa.

“(...) a veces el papá me ayuda y también me da una mano con la cocina”. (Entrevista 8 ,23 años, familia nuclear, no comparte la crianza, Centro de Salud 2)

Los efectores expresan también que la intervención del padre en las prácticas de cuidado no es muy activa, las mujeres madres le dejan al cuidado de sus hijo/as a una abuela o a una tía. 
“(...) la mayoría mamás, tengo abuelas, tengo papás, pero la mayoría son mamás y el nivel es al no ser tan bajo entienden y practican lo que uno le dice, (...)". (Entrevista Efector, Centro de Salud 2)

"Yo creo que es poco, yo creo que la intervención actual del papá no es muy activa, las mamás le dejan al cuidado de sus hijos a una abuela, a una tía." (Entrevista Efector, Centro de Salud 1)

Las mujeres se sitúan en el lugar de "cuidadoras" de la familia. El concepto de "cuidar de la familia, en especial a los chicos" es el núcleo central de la representación. Se ven como las "encargadas naturales" de los cuidados del cuerpo y de la alimentación, de transmitir los hábitos y la higiene cotidiana. La cultura ha propiciado el anclaje social que condiciona en la mujer un conjunto de comportamientos, creencias y actitudes con respecto al cuidado de los otros. La mujer se identifica y asume el rol de cuidar de los otros, en especial de apoyar y cuidar a los niños. Como expresa Jodelet, es importante conocer las funciones sociales que cumple la representación, debido a que permite entender cómo actúa la gente y cómo proceder ante esta situación, desde la responsabilidad del Estado $(11,27)$.

El término economía del cuidado se ha difundido de manera relativamente reciente para referir a un espacio bastante indefinido de bienes, servicios, actividades, relaciones y valores relativos a las necesidades más básicas y relevantes para la existencia y reproducción de las personas, en las sociedades en que viven (28). Esta visión permite identificar construcciones que perpetúan muchas de las inequidades existentes, como la creencia generalizada que sostiene que las mujeres están naturalmente mejor dotadas para llevar adelante el cuidado de los niños y niñas. Si bien no hay evidencias que sustenten este tipo de afirmaciones, siendo ésta diada mujer-cuidadora una construcción social, basada en las prácticas patriarcales hegemónicas (28).

\section{Discusión}

En el presente estudio hay coincidencia en lo que refieren cuidadoras y efectores en torno a la información brindada. Otro aspecto a tener en cuenta es la actitud de las cuidadoras en torno a la información recibida, en estos relatos aparecen los saberes previos que provienen de otras mujeres madres, que muchas veces entran en contraposición a la información provista por los efectores y que genera en las cuidadoras momentos de tensión que no se animan a verbalizar, ya que opinan que el médico es el que sabe. Al tomar la decisión, al interior de sus hogares, el saber que prevalece es el de otras mujeres madres. Esto concuerda con el trabajo de Candreva sobre las representaciones del cuidado a la salud en un grupo de mujeres, que encontró paradojas en el comportamiento, la mayoría de las mujeres reconocían tener información sobre determinados temas pero no poder actuar en consecuencia o no estar lo suficientemente convencidas para hacer aquello que sería beneficioso para la salud (27).

Los hallazgos del presente estudio, en relación a la comunicación unidireccional que se establece entre las cuidadoras y los efectores en el ámbito de la consulta, coinciden con un estudio realizado en Uruguay por Bathany que dice que el enfoque médico del cuidado, indagado a través de entrevistas a médicos pediatras, destaca la promoción de hábitos como elemento central del cuidado, despersonalizando el vínculo entre cuidador-niño, dejando la impresión de que la importancia del cuidador se basa en un saber que hay que transmitir al niño; no enfatiza en la singularidad de cada niño y en los procesos individuales, sino en la generalidades que presentan todos los niños según su edad (29). 
Otro hallazgo fue que las cuidadoras reconocen que no discuten con el médico sus prácticas o preferencias y los efectores aceptan que suelen dar información estereotipada, sin indagar si la misma es factible de poner en práctica o no por las cuidadoras. Esto concuerda con un estudio multicéntrico realizado en Argentina por Calvo, sobre nutrición en menores de 3 años, las madres de los niños describen a las instituciones de salud donde la respuesta está estereotipada, donde difícilmente encuentren una escucha atenta o una comunicación cálida, sin embargo es lo único que tienen. El mismo estudio de Calvo encontró que pocas madres discuten con el médico sus prácticas o preferencias. Desde los equipos de salud se favorece esta actitud pasiva de receptoras acríticas del saber del otro(30).

En esta investigación se encuentra en las cuidadoras contradicciones en algunas prácticas recomendadas por el equipo de salud con las recomendadas por su entorno. Ante esto, las cuidadoras no discuten ni confrontan, ya que opinan que el médico es el que sabe, esto concuerda la "Encuesta sobre Representaciones Sociales del Cuidado", que evidencia que si bien un tercio de la población consulta en primer lugar a sus madres sobre los aspectos de crianza, la cuarta parte de las mujeres consulta en primer lugar a los médicos o personal de la salud, lo que muestra el lugar de privilegio que tiene este saber en las prácticas y representaciones del cuidado de la salud (29).

El rol del equipo de salud se construye a través de un vínculo profesional de la saludcomunidad, en el que se generan expectativas de cumplimiento mutuo. En esta relación existe un sinnúmero de significaciones y condicionantes que se ponen en juego (económicos, políticos, etc.) que influyen en la concepción de que el médico es el que sabe y la comunidad es el objeto de conocimiento, tal como lo indican autores como Comes y Stolkiner, en un estudio realizado sobre las representaciones del derecho a la salud de un grupo de mujeres, reconocen que los programas de salud desarrollados en Argentina en la última década, conllevan en sus enunciados criterios de ciudadanía plena sin embargo entre sus acciones se encuentran prácticas nacidas del modelo asistencialista y caritativo (31).

Del análisis de las entrevistas, se visualiza que las mujeres madres se sitúan en el rol de cuidadoras y los hogares son el espacio natural donde se desarrolla el cuidado, también se advierte una escasa participación del varón en el cuidado y una constante tensión entre la información que reciben de los efectores y la que circula en sus redes de apoyo familiar. Estos hallazgos coinciden con un estudio realizado en Bs As, "cuidado de la salud: el anclaje social de su construcción (un estudio cualitativo)" donde se percibe que el cuidado de la salud se desarrolla de manera normada por la perspectiva de género, constituyéndose como un espacio social donde las mujeres priorizan la salud de los otros; encontrándose bien informadas respecto de causas, síntomas y mecanismos de prevención; y cuidado de la salud, pero los comportamientos y las prácticas no se corresponden con la información; lo que expresaría las rupturas que obstaculizan la adopción de prácticas de cuidados ante eventos concretos (27).

También coincide con un estudio realizado por Hernández Tezoquipa en México, quien concluye que las fuentes de conocimiento de las mujeres lo constituye su propia experiencia como pacientes o la desarrollada a través del cuidado a los miembros de la familia, la transmisión de conocimientos por abuelas, madres y compañeras y los medios masivos de comunicación (26).

Como se mencionó anteriormente, el cuidado tiene un rol femenino, con escasa participación del varón, coincidiendo con autores como Salas y Torre, que indican que los procesos de cuidados nutricionales en la infancia ocurren en un escenario de mandatos 
específicos para el desempeño femenino (32).

Diversos autores coinciden con el hallazgo de este estudio: en los sectores populares, el cuidado sigue siendo visto como responsabilidad de las mujeres, con los varones a veces "ayudando" en actividades que no reconocen como propias $(33,34)$.

Los resultados del presente estudio coinciden con autores como Jelin, Esquivel y Faur, quienes han identificado que en los sectores proveedores de cuidado, como educación, salud, y también el servicio doméstico; las mujeres se encuentran sobre representadas y persiste la idea de que las mujeres están "naturalmente" dotadas para proveer cuidados (35).También un estudio realizado por Salvia y Tunón, en Argentina coincide con el presente estudio: "en la práctica, la responsabilidad del cuidado infantil en la Argentina sigue siendo competencia principalmente de las mujeres madres. En efecto, la mayoría de los niños y niñas menores de 5 años pasan la mayor parte de su tiempo con su madre" (36). De los relatos de las cuidadoras, se aprecia que ellas se reconocen como tales, y en quienes refieren compartir el cuidado, éste es aportado por otras mujeres madres (abuelas), coincidiendo con la encuesta de uso del tiempo realizada en Uruguay, que muestra que cuando hay niños en el hogar las mujeres son las cuidadoras principales (37); y con un estudio realizado en Colombia sobre patrones de crianza y cuidados en niños y niñas, se encontró que éstos eran considerados una cuestión de mujeres; labor que ha estado implícita en los denominados oficios domésticos (38). También un estudio realizado sobre percepciones y prácticas alimentarias de un grupo de mujeres de poblaciones rurales de México, encontró coincidencias en las respuestas de las mujeres, ellas opinan que así como los hombres tienen que salir a trabajar fuera de la casa, las mujeres deben responsabilizarse de lo que ocurre en el hogar (32).A su vez, Ariza y Olivera concluyen que al interior del hogar los niños son cuidados no solo por las madres y empleadas domésticas, y marginalmente por los padres. Las hijas mayores, las abuelas y otras parientas tienen un rol importante. Este aporte se enmarca en relaciones de cooperación, intercambio y negociación al interior de las familias, relaciones que son fundamentalmente de carácter jerárquico porque siguen la distribución de poder que marcan dos ejes de diferenciación social: el género y la generación (39).

\section{Conclusiones}

Los actores entrevistados fueron por un lado, profesionales que se desempeñan en los programas analizados, en su mayoría mujeres; y por otro, cuidadoras nutricionales que asisten a dos centros de salud municipales, en su totalidad mujeres madres, que conforman familias nucleares o extendidas, el $60 \%$ no trabaja y el $60 \%$ comparte la crianza con otras mujeres.

Las representaciones de los efectores están dominadas por el modelo hegemónico imperante en salud; caracterizada por acciones como informar, prohibir y disponer de acciones normativas. Las cuidadoras se sitúan en el rol de cuidadora, como mandato natural por ser mujer.

Las cuidadoras en sus relatos solo aceptan las recomendaciones y reconocen los conocimientos del médico, aunque luego en la práctica, prevalece el saber adquirido en la vida, en la experiencia de las abuelas y otros familiares, no sin sentirse descalificadas por el equipo de salud, por lo que prefieren quedarse en un rol pasivo en la consulta.

La comunicación entre las cuidadoras y los efectores se desarrolla en un escenario marcado por la hegemonía, las cuidadoras reconocen los conocimientos del médico, no cuestionando las indicaciones recibidas, teniendo una actitud pasiva como receptora acrítica, lo que muestra el lugar de privilegio que tiene el saber académico en las prácticas y representaciones del cuidado de la salud; pero que les genera conflictos al interior de la familia al momento de ponerlos en práctica. Los profesionales de salud reconocen en 
sus relatos que la comunicación con las cuidadoras es unidireccional, ellas toman esta información brindada como una consejería, confiando en el saber que los profesionales poseen, y a su vez los profesionales no hacen un auto reflexión acerca de la consejería como un acto dialógico.

Si entendemos el cuidado como uno de los factores subyacentes que determinan la ingesta de nutrientes y la salud, y por lo tanto, un factor determinante en el proceso de crecimiento y desarrollo de niños y niñas; debemos reconocer que todos los seres humanos contamos con un legado de creencias y prácticas de cuidados que son heredados de generación en generación, esto merece ser considerado para no desconocer y desaprovechar el conocimiento de los sujetos. Por lo que resulta imperante capacitar a los equipos de salud en temas referidos al cuidado nutricional infantil, pero sin despersonalizar el vínculo entre cuidador-niño, e incluyendo la afectividad como aspecto central.

Sería necesario, si se propone abordar a una construcción del ejercicio pleno de los derechos, analizar los procesos de implementación e interpretación de los programas para que incorpore la participación ciudadana y su real protagonismo en la construcción colectiva, para esto es necesario transitar del modelo hegemónico hospitalocéntrico a otro, para que los profesionales se acerquen y trabajen con la comunidad, proponiendo acciones de promoción y prevención desde ella

\section{Bibliografía}

1. Barbero L., Martina D., Scruzzi G., Iavicoli T., Pérez M. Promoción de buenas prácticas de cuidado nutricional infantil. Entrecruzando docencia, extensión e investigación. 2012. [En línea] Disponible en: http://www.agro.uncor. edu/ paginafacu/FORO/Archivos/Articulacion\%20Entre\%20Docencia, \%20 Investigacion $\% 20 \mathrm{y} \% 20$ Extension/54Martina.pdf

2. Freier, S. Universidad Católica Argentina. Instituto para la Integración del Saber Departamento de Investigación Institucional. Área Política "Las políticas sociales comparadas entre los años ' 60 y '90 en Argentina”. 2002. [En línea]. Disponible en: http://www.uca.edu.ar/uca/common/grupo32/files/freier3-2002.pdf

3. Comas D’Argemir, D. Mujeres, familia y estado del bienestar. En del Valle, Teresa (Editora) Perspectivas feministas desde la Antropología Social. Barcelona: Ariel, 2000, p 187-204

4. Engle, P., Menom, P., Haddad, L. Care and nutrition. Concepts and measurement. [En lineal]. FCND DiscussionPaper No 18. IFPRI. August 1996. Disponible en la URL: www.ifpri.org/DIVS/FCND/dp/papers/dp18.pdf [Consulta: Febrero 2012].

5. Latham, M. Parte V. Políticas y Programas de Nutrición. Capítulo 36. Cuidados y Nutrición. En su: Nutrición Humana en el Mundo en Desarrollo. Colección FAO. Alimentación y Nutrición No 29. FAO. 2002. p 369-377

6. Letablier M.T. "El trabajo de 'cuidados' y su conceptualización en Europa". En: Prieto (ed.) (ed.) Trabajo, género y tiempo social. Hacer/Complutense, Madrid. 2007.

7. Aguirre R. "Los cuidados familiares como problema público y objeto de políticas" En Arriagada, I. Familias y políticas públicas en América Latina: Una historia de desencuentros, Comisión Económica para América Latina y el Caribe (CEPAL), Santiago de Chile.2007

8. Pautassi L "El cuidado como cuestión social desde un enfoque de derechos". Serie Mujer y Desarrollo. CEPAL. Unidad Mujer y Desarrollo. Octubre 2007.

9. Jelin E. Pan y afectos. La transformación de las familias, Fondo de Cultura Económica, Buenos Aires. 2010

10. Ley Nacional 26.061 Boletín Oficial. Argentina, 21 de octubre de 2005.

11. Jodelet D. "La representación social: fenómenos, concepto y teoría". En: Moscovici, 
Serge (Ed.) "Psicología social II. Pensamiento y vida social. Psicología social y problemas sociales." Paidos, Buenos Aires. 1986.

12. Araya Umaña S. Las Representaciones Sociales. Ejes teóricos para su discusión. Facultad Latinoamericana de ciencias sociales (FIACSO) Sede académica Costa Rica, 2002.

13. Botero, P. Representaciones y ciencias sociales una perspectiva epistemológica y metodológica. Espacio Editorial, Buenos Aires. Argentina. 2008. P 88-107.

14. Mendizábal N. Capítulo 2. Los componentes del diseño flexible en investigación cualitativa. En: Vasilachis de Gialdino I. Estrategias de Investigación Cualitativa. Editorial Gredisa. Biblioteca de Educación. Noviembre 2006.

15. Mora M. La teoría de las representaciones Sociales de Sergei Moscovici. Universidad de Guadalajara. Athenea digital. México 2002. [En línea] Disponible en: http:// www.academia.edu/214897/La_Teoria_de_las_Representaciones_Sociales_de_ Serge_Moscovici

16. Glaser B., Strauss A. The discovery of grounded theory: strategies for qualitative research. New York: Aldine Publishing Company. Capítulo 5: "El método de comparación constante de análisis cualitativo". 1967.p. 101-115.

17. Municipalidad de Córdoba. Secretaria de Salud. Dirección de atención primaria a la salud. Programa de control de crecimiento y desarrollo del niño. [Monografía DVD]. Tercera comisión de revisión 2010)

18. Melguizo Herrera E, Alzate Posada ML. Creencias y prácticas en el cuidado de la salud. Avances en Enfermería, 2008, vol. 26, no 1, p. 112-123

19. Aguiló Pastrana E. La metodología de los procesos correctores comunitarios (ProCC). Revista clínica electrónica en Atención Primaria, 2008, no 16, p. 0001-5.

20. De la Vega, SM. La invisibilización del cuidado en la familia y los sistemas sanitarios. Política y sociedad, 2000, vol. 35, p. 73

21. Sánchez Arrastia D, Contreras Olivé Y. La relación médico-paciente y su importancia en la práctica médica. Revista Cubana de Medicina Militar, 2014, vol. 43, no 4, p. 528-533.

22. Moore P, et al. La comunicación médico paciente: ¿Cuáles son las habilidades efectivas? Revista médica de Chile, 2010, vol. 138, no 8, p. 1047-1054.

Artaza O, Sagastuy B. Construyendo una estrategia para la cobertura universal en salud. [en línea] Disponible en http://boletin.ciess.org/boletines/2014/6/pdf/ Construyendo_una_estrategia_para_la_cobertura_universal_e_salud.pdf

23. Llobet V. Las políticas sociales para la infancia vulnerable. Algunas reflexiones desde la Psicología. Rev LatinoamCiencSocNiñezJuv [Internet]. 2011 [cited 2014 Jul 27]; 4(1). Available from: http://revistaumanizales.cinde.org.co/index.php/ Revista-Latinoamericana/article/viewArticle/391

24. Ministerio de Salud de Argentina. Guia de Comunicación para equipos de salud. Serie Comunicación y salud desde una perspectiva de derechos. Edición $\mathrm{N}^{\circ} 2$ (Septiembre 2015) Buenos Aires. Disponible en: http://www.msal.gob.ar/planreduccion-mortalidad/descargas/guia-comunicacion.pdf

25. Hernandez M, Tezoquipa L, Arenas ML., Santiago RV. El cuidado a la salud en el ámbito doméstico: interacción social y vida cotidiana. RevSaúde Pública, 2001, vol. 35, no 5, p. 443-50

26. Candreva A, Paladino C. Cuidado de la salud: el anclaje social de su construcción estudio cualitativo. Universitaspsychologica, 2005, vol. 4, no 1, p. 55-62.

27. Rodríguez Enriquez C. Economía del cuidado y política económica. Una aproximación a sus interrelaciones. En documento presentado a la trigésimo octava reunión de la Mesa Directiva de la Conferencia Regional sobre la Mujer de América Latina y el Caribe, Comisión Económica para América Latina y el Caribe (CEPAL), septiembre. 2005.

28. Batthyany, k. Genta, N. Perrotta, V. La población uruguaya y el cuidado: Persistencias de un mandato de género. Encuesta nacional sobre representaciones 
sociales del cuidado: Principales resultados. 2012. [En línea] Disponible en: http:/www.cepal.org/publicaciones/ $\mathrm{xml} / 6 / 48256 /$ SLapoblacionUruguayayelcuidado.pdf.

29. Calvo E y col. Estudios sobre nutrición en menores de 3 años, alimentación y riesgo de desnutrición: becas Ramón Carrillo-Arturo Oñativia, estudio colaborativo multicéntrico año 2003. En Estudios sobre nutrición en menores de 3 años, alimentación y riesgo de desnutrición: becas Ramón Carrillo-Arturo Oñativia, estudio colaborativo multicéntrico año 2003. Comisión Nacional de Programas de Investigación Sanitaria, 2005.

30. Comes Y, Stolkiner A. Representaciones sociales del derecho a la atención de la salud de un grupo de mujeres pobres. Anuario de investigaciones, 2006, vol. 13, p. 211219.

31. Bertran M, Arroyo P. Para la Salud FM. Antropología y nutrición [Internet]. Fundación Mexicana para la Salud; 2006 [cited 2015 Jul 17]. Available from: http:// www.dimensionantropologica.inah.gob.mx/wpcontent/ uploads/07Rese \%C3\%B1as43.pdf

32. Martínez Franzoni J, Voorend K. Desigualdades de género en los regímenes de bienestar latinoamericanos. En: Laura Pautassi y Carla Zibecchi (comp.) Las fronteras del cuidado. Agenda, derechos e infraestructura. Buenos Aires: editorial Biblos-ELA.[Links], 2013.

33. Faur E. Género y conciliación familia-trabajo: legislación laboral y subjetividades masculinas en América Latina. Cohesión social, políticas conciliatorias y presupuesto público, una mirada desde el género, 2006.

34. Esquivel V, Faur E, Jelín E. Hacia la conceptualización del cuidado: familia, mercado y Estado. Las LógCuidInfant Entre Las Fam El Estado El Merc. 2012;11-43.

35. Tuñón I, Salvia A. La deuda social con la niñez y adolescencia: Magnitud, evolución y perfiles [Internet]. Barómetro de la Deuda Social de la Infancia, Observatorio de la Deuda Social Argentina. Buenos Aires: Fundación UCA-Arcor; 2010 [cited 2014 Sep 26]. Available from:http://bibliotecadigital.uca.edu.ar/repositorio/ investigacion/deuda-social-ninez-adolescencia-magnitud. pdf

36. Aguirre R; Batthyány K. El cuidado infantil en Montevideo. Análisis de resultados de la encuesta sobre uso del tiempo: desigualdades sociales y de género, Universidad de la República, UNICEF, Montevideo, Uruguay. 2005.

37. Triana A, Avila L y Malagón A. Patrones de crianza y cuidados en niños y niñas de Boyacá. Rev. Latinoamericana de Cs. Sociales, Niñez y Juventud. 8(2):933-945. 2010.

38. Ariza, M. y De Oliveira O. Acerca de las familias y los hogares: estructura y dinámica. En: Catalina Wainerman (compiladora). Familia. Trabajo y Género: Un mundo de nuevas relaciones. Fondo de las Naciones Unidas para la Infancia (UNICEF) y Fondo de Cultura Económica de Argentina, 2003. 